\title{
Retirement age and health expenditures ${ }^{1}$
}

\author{
Helmuth Cremer ${ }^{2}$, Jean-Marie Lozachmeur ${ }^{3}$ \\ and Pierre Pestieau ${ }^{4}$
}

September 2004

\footnotetext{
${ }^{1}$ This paper has been presented at the 3rd Journées d'Economie Publique Louis-André Gérard-Varet. We would like to thank the participants and in particluar our discussant, Alain Trannoy, for their comments and suggestions. Support for this reasearch project has been provided by the European RTN program through the FINRET network.

${ }^{2}$ Corresponding author: GREMAQ and IDEI, University of Toulouse, 21 Allée de Brienne, 31000 Toulouse, France; e-mail: helmut@cict.fr.

${ }^{3}$ GREMAQ, University of Toulouse.

${ }^{4}$ CREPP, Université de Liège, CORE, Université Catholique de Louvain and Delta.
} 


\begin{abstract}
This paper studies the design of pension benefits and contributions when an individual's health status (disutility of continued activity) is endogenous and depends on his consumption of health services. Health services can be subsidized (in a linear or non linear way, depending on the information structure). Uniform public provision of health services is also considered. We show that like with exogenous health status, the secondbest policy may induce early retirement for some types of individuals. Furthermore, whatever the specific information structure considered (individual levels or anonymous transactions) for health services, a subsidization of health expenditures obtains under fairly plausible assumptions. Third, when the information structure permits only linear subsidies, the case for uniform public provision of health services (which can be supplemented but not resold) appears to be quite strong.
\end{abstract}




\section{Introduction}

Over the last decade the future of pension systems has been a widely debated issue. The challenges posed by the ongoing process of demographic ageing are by now understood. However, aging is only part of the story. The generalized decrease of entry ages into retirement is also a significant problem. There are a number of explanations for this trend. The dominant one is the implicit taxation on continued activity of elderly workers. This argument is based on the idea that workers have, individually or collectively, a large degree of discretion concerning retirement age. In that line, Gruber and Wise $(1999,2002)$ have shown for a dozen of OECD countries that households often face very powerful financial incentives pushing them to retire early.

However early retirement policies that come from various sources, early retirement programs but also unemployment and disability insurance schemes, cannot be easily abandoned. They also serve the objective of helping those who are unable to work long because of disability or don't want to work long because of low productivity. Unfortunately, these redistribution and insurance objectives cannot be easily fulfilled because productivity and health are typically not observable. It is thus important to design a system of retirement and disability insurance which takes those informational constraints into account. Our earlier papers Cremer et al (2004a-c) have studied this problem under specific assumptions. They have shown that the optimal second best policy may imply distortions like early retirement or exit from the labor force of healthy workers through disability insurance schemes. While these papers consider settings in which individuals differ in "health status", they have considered this source of heterogeneity as purely exogenous. ${ }^{1}$ However, in reality an individual's health status may be, at least in part, endogenous. In particular it may be affected by his consumption of health services. This additional ramification brings about a number of interesting questions. First, one may wonder whether the results obtained with exogenous health

\footnotetext{
${ }^{1}$ In Cremer et al. (2004a), health status (along with other adverse selection variables like productivity) is not obsevrable. The possibility of costly heatlh testing (auditing) is considered in Cremer et al. (2004b). Cremer et al. (2004c) explicitly introduce the distinction between disabled individuals on the one hand, and leisure prone individuals, on the other hand. While both of these two types of individuals have a hight disutility of labor, they may not be accounted for in the same way in social preferences.
} 
stand in such a more general setting. Second, there is the issue of public intervention in the health sector. Specifically, should public policy interfere with individuals' choice of health service consumption (through subsidization or public provision)? Third, there is the more fundamental question of whether extra resources should be expended to make individuals more "equal" or whether it is more appropriate to use this money to compensate the less healthy individuals. ${ }^{2}$

This paper addresses these questions by studying the design of a social security scheme which can be supplemented by health policies including subsidization of health expenditures and public provision of health care. We consider a setting where agents differ both in their productivity and in their health status. The health status determines the disutility of intensive (work week) and extensive (career length) work. Additionally, it is also affected by health expenditures. Thus, an increase in health expenditures can lower the disutility of work. Social preferences are assumed to be utilitarian and neither the health status nor the productivity of individuals are publicly observable. The optimal policy is represented by a tax/transfer function which depends on labor income, the age of retirement and the health expenditures (if observable). We first present the optimum when the government can observe individuals' health expenditures and then turn to the case where health expenditures are anonymous transactions. Finally, we consider the case for public provision of health services as additional instrument which is used along with an optimal tax policy.

\section{The model}

The model we use is a reduced form of an intertemporal model. It is inspired by the specification used in Cremer et al. (2004a) who also discuss its micro foundations. ${ }^{3}$ Consider an individual whose length of life is normalized at 1 and whose consumption level, $c$, is constant over time. His labor supply is constant at level $l$ up to $z$ (with $0 \leq z \leq 1)$ and equal to zero afterwards. For concreteness we shall often refer to $l$

\footnotetext{
${ }^{2} \mathrm{~A}$ similar issue arises when individuals earning ability (productivity) depends on their education; see e.g., Cremer and Pestieau (2004) and Maldonado (2004).

${ }^{3}$ Monotonicity conditions are also discussed in detail there. We only recall those which are directly relevant for the problem considered here.
} 
as "weekly labor supply" while $z$ is interpreted as "retirement age". Preferences are represented by the following (reduced form) utility function

$$
u(c)-V(l) R(z, H)
$$

The function $R(z, H)$ denotes the disutility for a working life of length $z$ with $R_{z}>0$ and $R_{z z}>0 .{ }^{4}$ Labor disutility, regarding the length of working life $z$, can be interpreted in terms of an indicator of health status. Healthy individuals accordingly would have a lower $R$ than individuals whose poor health makes it harder to work beyond a certain age. An individual's health status also depends on his consumption of health services $H \geq 0$, where $R_{H}<0$ and $R_{H H}>0$. The producer prices of health services is constant and normalized at 1 . There is another term in the labor disutility which concerns the length of work week, $V(l) .{ }^{5}$ In the laissez-faire an individual's budget constraint is given by $c+H=D=z w l$ where $D$ is the disposable income.

Individuals differ in their productivity, $w$ and in their health status $R$. For simplicity we consider only two types of individuals, indexed $i=1,2$, with $w^{2} \geq w^{1}, R^{2}(z, H) \leq$ $R^{1}(z, H)$ for all $z \in[0,1]$ and $H \geq 0$. In words, type 2 is the "good" type who is both more productive and more healthy (his disutility of continued activity is smaller). ${ }^{6}$

Some of our results below rely on a more specific assumption:

Assumption 1 We have $(i) R_{z}^{2}(z, H) \leq R_{z}^{1}(z, H)$ and $(i i) R_{H}^{1}(z, H) \leq R_{H}^{2}(z, H)$ for all $z \in[0,1]$ and $H \geq 0$.

The first of these conditions states that type 2's disutility of continued activity

\footnotetext{
${ }^{4}$ Unless otherwise stipulated, subsripts stand for partial derivatives.

${ }^{5}$ As discussed by Cremer et al. (2004) the reduced form function (1) can be obtained under some assumptions from the maximization of lifetime utility with an instantaneous utility function

$$
U(t)=u(c(t))-r(t, H) V(l(t)) .
$$

For instance, when $c(t)$ is constant (interest rate equal rate of time preference) and when we set $l(t)=l$ for $t \leq z$ and 0 afterwards, we can define

$$
R(z, H)=\int_{0}^{z} r(t, H) d t
$$

${ }^{6}$ We assume that the term $V(l)$ is the same for everyone. When returning to the micro foundations discussed in footnote 5 one can easily show that this assumption does not impose any additional restrictions. Any heterogenity in labor disutlity can be subsumed in $r(t, H)$.
} 
which by definition is smaller than that of type 1 , also increases at a lower rate. To interpret $(i i)$, recall that $R_{H}<0$. It is therefore convenient to rewrite the condition in terms of positive numbers yielding $-R_{H}^{1}(z, H) \geq-R_{H}^{2}(z, H)$. Assumption 1 (ii) thus states that (for given retirement age and health expenditure), an increase in health expenditures induces a larger reduction in $R$ for the individual with the poorer health (namely individual 1).

Throughout the paper we shall assume that an individual's ("weekly") income $y=w l$, and the retirement age $z$ are observable while $w, l$ and $R$ are not observable. Neglecting $H$ for the time being, the tax cum retirement policy can be summarized in a net tax-transfer function $T(y, z)$ which depends on the observable variables. We have studied the design of this function in Cremer et al. (2004a). Specifically, we have examined whether the retirement decision (choice of $z$ ) ought to be distorted in a second best setting; i.e., whether it is desirable to impose a so called "implicit tax on continued activity". In the current paper we continue to assume that the tax and retirement policies are designed in a optimal way but we concentrate on the public policies involving health services. We shall examine whether the optimal policy mix ought to include a subsidy of health expenditures or possibly a public provision of health services. We look at this issue under two informational assumptions. First, we assume that individual consumption levels of health services are publicly observable. In that case, subsidies of health expenditures can take a very general form. In particular they can be nonlinear and conditional on income (means tested). Then, we assume that only anonymous transactions are observable so that only linear subsidies on health expenditures are available. ${ }^{7}$

\section{Publicly observable personal consumption of health ser- vices}

\subsection{Policy instruments and individual choices}

When $H$ is observable at the individual level, it can be used as an argument in the net transfer function $T(y, z, H)$. Now this function specifies not only the individual

\footnotetext{
${ }^{7}$ For a more detailed discussion of the impact of the information structure on the set of feasible instruments, see Cremer and Gahvari (1997).
} 
retirement benefits (net of payroll taxes) but also any (positive or negative) subsidy of health expenditures. With such a transfer function individuals solve the following problem:

$$
\begin{array}{ll} 
& \underset{c, z, l, H}{ } \operatorname{Max} u(c)-V\left(\frac{y}{w}\right) R(z, H) \\
\text { s.t. } \quad: \quad & c=z y-H-T(y, z, H)
\end{array}
$$

Substituting (3) into (2) and differentiating with respect to the remaining variables yields the following first-order conditions with respect to $y, z$, and $H$ :

$$
\begin{aligned}
w\left(z-T_{y}\right) u^{\prime}(c)-V^{\prime}(l) R(z, H) & =0 \\
\left(y-T_{z}\right) u^{\prime}(c)-V(l) R_{z}(z, H) & =0 \\
-\left(1+T_{H}\right) u^{\prime}(c)-V(l) R_{H}(z, H) & =0
\end{aligned}
$$

Observe that by setting $T(y, z, H) \equiv 0$ (so that $T_{y}=T_{z}=T_{H}=0$ ) we obtain the laissezfaire solution at which individuals' choices are not distorted. Since we are interested in the determination of $z, H$ and the interaction between $H$ and $z$, we concentrate on equations (6) and (5) which can be rewritten as follows:

$$
\begin{aligned}
M R S_{c z} & =\frac{V(l) R_{z}(z, H)}{u^{\prime}(c)}=y-T_{z} \\
M R S_{c H} & =-\frac{V(l) R_{H}(z, H)}{u^{\prime}(c)}=\left(1+T_{H}\right), \\
M R S_{H z} & =-\frac{R_{z}(z, H)}{R_{H}(z, H)}=y\left[\frac{1-\frac{T_{z}}{y}}{1+T_{H}}\right]=y[1-\theta]
\end{aligned}
$$

where $M R S_{c z}$ is the marginal rate of substitution between $z$ and $c$ and $M R S_{c H}$ is the marginal rate of substitution between $H$ and $c$ (i.e. the slope of an indifference curve in the $(H, c)$ space). Similarly, $M R S_{H z}$ is the marginal rate of substitution in the $(z, H)$ space while $\theta$ is defined as

$$
\theta=\frac{T_{H}+\frac{T_{z}}{y}}{1+T_{H}}
$$

Not surprisingly, condition (8) states that the marginal rate of substitution between $H$ and the numeraire be equal to the net marginal price of $H$, i.e., the producer price 
minus or plus any subsidy or tax. When $T_{H}<0$ we have a marginal subsidy and the net price of $H$ is less than 1. Similarly, from (7), there is a marginal tax on continued activity when $T_{z}>0$.

Equation (9) describes the tradeoff between health expenditures and retirement age. This is understood most easily by looking at the "effort minimization" problem. This is the "dual" problem of utility maximization in the sense that to maximize utility (2) subject to the budget constraint (3) it is necessary to minimize "effort" defined by $E=V(l) R(z, H)$ for a given level $I$ of life-cycle income net of health expenditures (i.e., subject to $I=w l z-T(y, z)-p H$, where $I$ is exogenously given and set at the appropriate level). This problem is represented on Figure 1 in the $(z, H)$ space for a given level of $y$. The solution is a point of tangency between an "iso-effort curve" and the income constraint. When there are no taxes or subsidies, $\left(T_{z}=T_{H}=0\right)$ the slope of the income constraint is $y$. When marginal tax rates are not zero, the slope equals $y(1-\theta)$. When $\theta<0$ (i.e. when the subsidy on health, $-T_{H}$ exceed $T_{z} / y$ ) the slope is larger than $y$ and $H$ is encouraged with respect to $z$. A positive $\theta$ on the other hand implies a distortion in favor of $z .^{8}$

An alternative view on these distortions consists in saying that the choice between $H$ and $z$ is distorted upward $(\theta<0)$ if individuals who increase their health expenditures pay less taxes, for a given level of before tax lifetime income $I=y z-H$ (i.e., when $z$ is determined by $z=(I+H) / y)$ and a given $y$. Denoting $T T(H)=T(y,(I+H) / y, H)$ the total tax paid by an individual, this variation is given by

$$
\frac{d T T(H)}{d H}=T_{H}+\frac{T_{z}}{y}
$$

which has the same sign of $\theta$.

\subsection{Second best optimum}

We proceed by characterizing the (utilitarian) Pareto efficient allocation constrained by the information structure. Denoting $f^{i}$ the proportion of type $i$ individual, the Lagrangian expression associated with this problem is given by

\footnotetext{
${ }^{8}$ The distortions mentioned here are substitution effects, for given levels of $I$.
} 


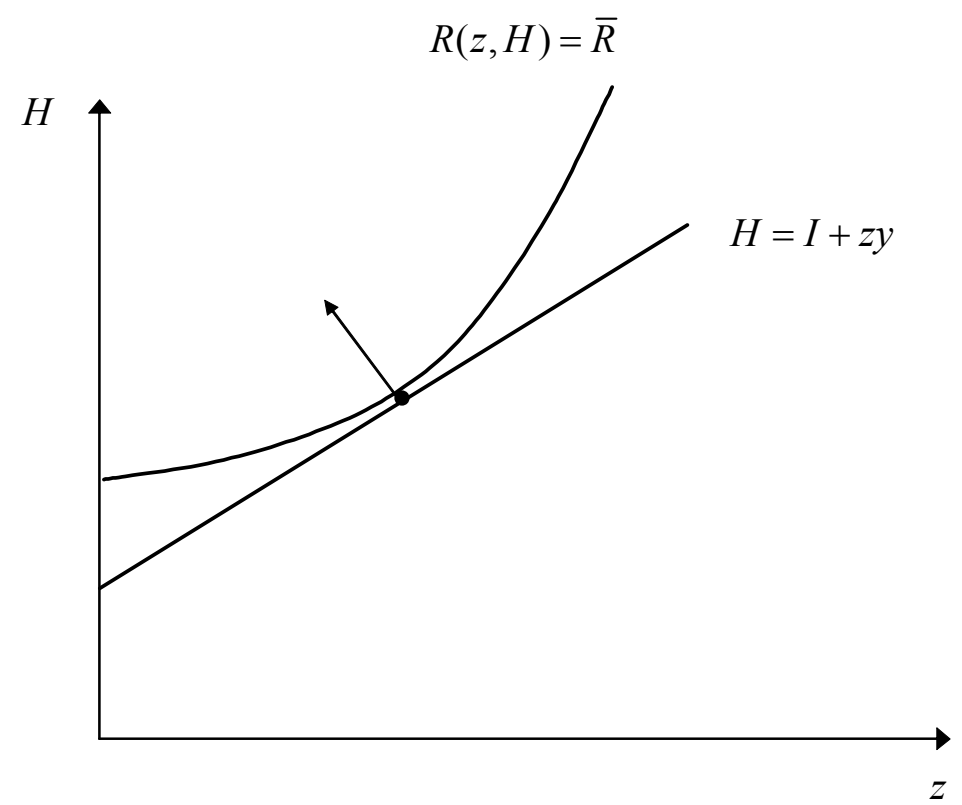

Figure 1: Effort minimization and distortions in the $(z, H)$ space. 


$$
\begin{aligned}
& \max _{c^{i}, y^{i}, z^{i}, H^{i}} \sum_{i} f^{i} U^{i}+\mu\left(\sum_{i} f^{i}\left(y^{i} z^{i}-c^{i}-H^{i}\right)\right) \\
& +\lambda\left(u\left(c^{2}\right)-V\left(\frac{y^{2}}{w^{2}}\right) R^{2}\left(z^{2}, H^{2}\right)-u\left(c^{1}\right)+V\left(\frac{y^{1}}{w^{2}}\right) R^{2}\left(z^{1}, H^{1}\right)\right)
\end{aligned}
$$

where $\mu>0$ is the multiplier of the resource constraint, while $\lambda$ is the multiplier associated with the "downward" self-selection constraint (from the productive and healthy type 2 to the less productive and less healthy type 1). As usual in utilitarian case, this downward incentive constraint is necessarily binding so that we have $\lambda>0$. The first-order conditions with respect to $c^{i}, z^{i}, H^{i}(i=1,2)$ are given by

$$
\begin{aligned}
& f^{1} u^{\prime}\left(c^{1}\right)-\mu f^{1}-\lambda u^{\prime}\left(c^{1}\right)=0, \\
& f^{2} u^{\prime}\left(c^{2}\right)-\mu f^{2}+\lambda u^{\prime}\left(c^{2}\right)=0, \\
& -f^{1} V\left(l^{1}\right) R_{z}^{1}\left(z^{1}, H^{1}\right)+\mu f^{1} y^{1}+\lambda V\left(\bar{l}^{2}\right) R_{z}^{2}\left(z^{1}, H^{1}\right)=0, \\
& -f^{1} V\left(l^{2}\right) R_{z}^{2}\left(z^{2}, H^{2}\right)+\mu f^{2} y^{2}-\lambda V\left(l^{2}\right) R_{z}^{2}\left(z^{2}, H^{2}\right)=0, \\
& -f^{1} V\left(l^{1}\right) R_{H}^{1}\left(z^{1}, H^{1}\right)-\mu f^{1}+\lambda V\left(\bar{l}^{2}\right) R_{H}^{2}\left(z^{1}, H^{1}\right)=0, \\
& -f^{1} V\left(l^{2}\right) R_{1 H}\left(z^{1}, H^{1}\right)-\mu f^{2}-\lambda V\left(l^{2}\right) R_{H}^{2}\left(z^{2}, H^{2}\right)=0,
\end{aligned}
$$

where $\bar{l}^{2}=y^{1} / w^{2}$ is the (weekly) labor supply of the "mimicking" individual. ${ }^{9}$

Combining (13) and (17) yields $M R S_{c H}^{2}=1$, which along with (8) implies $T_{H}^{2}=0$ : there is no subsidy (or tax) on individual 2's consumption of health services. This is the usual "no distortion at the top result", which of course also applies to $z$ and $y$.

For individual 1, combining (12) and (14) yields

$$
M R S_{c z}^{1}=y_{1}\left[\frac{f^{1}-\lambda}{f^{1}-\lambda \frac{\overline{M R S_{c z}^{2}}}{M R S_{c z}^{1}}}\right],
$$

where

$$
\frac{\overline{M R S}_{c z}^{2}}{M R S_{c z}^{1}}=\frac{V\left(\bar{l}^{2}\right) R_{z}^{2}\left(z^{1}, H^{1}\right)}{V\left(l^{1}\right) R_{z}^{1}\left(z^{1}, H^{1}\right)}<1 .
$$

\footnotetext{
${ }^{9}$ The solution must of course also satisfy the first-order conditions with respect to $y_{i}$. We have omitted these expressions because we make no explicit use of them in our subsequent arguments.
} 
This implies $M R S_{c z}^{1}<y_{1}$ so that there is a marginal downward distortion in the retirement age. In other words, and making use of equation (7), the marginal tax on the age of retirement, $T_{z}$, is positive. Consequently, it appears that the main result of Cremer et al. (2004) continues to hold when the disutility of continued activity is endogenous and depends on the consumption of health services.

Equivalently, combining (12) and (16) yields

$$
M R S_{c H}^{1}=\left[\frac{f^{1}-\lambda}{f^{1}-\lambda \frac{\overline{M R S}_{c H}^{2}}{M R S_{c H}^{1}}}\right],
$$

where

$$
\frac{\overline{M R S}_{c H}^{2}}{M R S_{c H}^{1}}=\frac{V\left(\bar{l}^{2}\right) R_{H}^{2}\left(z^{1}, H^{1}\right)}{V\left(l^{1}\right) R_{H}^{1}\left(z^{1}, H^{1}\right)} .
$$

From the comparison of (19) and (8), it follows that the sign of $T_{H}^{1}$ depends on whether $M R S_{c H}^{1}$ is smaller of larger than one. To examine this we start by studying the RHS of (20). With $w^{2} \geq w^{1}$ we have $V\left(\bar{l}^{2}\right) \leq V\left(l^{2}\right)$. Furthermore, Assumption 1 implies $R_{H}^{2}\left(z^{1}, H^{1}\right) \leq R_{H}^{1}\left(z^{1}, H^{1}\right) \cdot{ }^{10}$ Consequently we have

$$
\frac{\overline{M R S}_{c H}^{2}}{M R S_{c H}^{1}} \leq 1
$$

which from (19) implies $M R S_{c H}^{1} \geq y$ and thus $T_{H}^{1} \leq 0$. Observe that to obtain a strict inequality it is sufficient to have either $w^{2}>w^{1}$ or to have a strict inequality in Assumption 1. In other words, health expenditures of the type 1 individual ought to be subsidized at the margin. Most significantly, the use of this instrument improves over the situation where only income taxation is used. This result is intuitively quite appealing. In our setting health expenditures are an effective way of redistributing from the productive and healthy to the less productive and less healthy. As usual in optimal tax model, redistribution is limited by self-selection and we have to find a form of redistribution which is more attractive to type 1 individuals than to type 2 individual (who considers mimicking type 1). This is exactly what the subsidization of $H$ achieves, for the willingness to pay for $H$ of a type 1 individuals is higher than that

\footnotetext{
${ }^{10}$ Recall that $R_{H}<0$ so that $R_{H}^{2}\left(z_{1}, H_{1}\right) \leq R_{H}^{1}\left(z_{1}, H_{1}\right)$ implies $R_{H}^{2}\left(z_{1}, H_{1}\right) / R_{H}^{1}\left(z_{1}, H_{1}\right) \leq 1$.
} 
of a mimicking type 2 individual. Observe that the special case where $M R S_{c H}^{1}=1$ occurs when $w^{2}=w^{1}$ and $R_{H}^{2}\left(z^{1}, H^{1}\right)=R_{H}^{1}\left(z^{1}, H^{1}\right)$. In this case, individual 1 and 2 indifference curves in the $(c, H)$ space have the same slope so that a distortion cannot relax an otherwise binding self-selection constraint.

We now turn to the tradeoff between health and retirement age. Not surprisingly, there is no distortion for individuals 2. To see this, combine (13) and (15) to show $M R S_{H z}^{2}=y^{2}$ which, along with (9), implies $\theta_{2}=0$. Turning to individual 1, combining (16) and (14), we have

$$
M R S_{H z}^{1}=\frac{f^{1}-\lambda \frac{\overline{M R S}_{c H}^{2}}{M R S_{c H}^{1}}}{f^{1}-\lambda \frac{\overline{M R S_{c z}^{2}}}{M R S_{c z}^{1}}} y^{1},
$$

where

$$
\frac{\overline{M R S}_{c z}^{2}}{M R S_{c z}^{1}}=\frac{V\left(\bar{l}^{2}\right) R_{z}^{2}\left(z^{1}, H^{1}\right)}{V\left(l^{1}\right) R_{z}^{1}\left(z^{1}, H^{1}\right)},
$$

while $\overline{M R S}_{c H}^{2} / M R S_{c H}^{1}$ continues to be given by (20). Rearranging the RHS of (21) while using (22), (20) and defining $\varepsilon_{z}^{i}$ and $\varepsilon_{H}^{i}$ as individual $i$ 's $(i=1,2)$ elasticity of $R$ with respect to $z$ and $H$ respectively:

$$
\varepsilon_{z}^{i}=\frac{R_{z}^{i}(z, H) z}{R^{i}(z, H)} \quad \varepsilon_{H}^{i}=\frac{R_{H}^{i}(z, H) H}{R^{i}(z, H)},
$$

yields:

$$
M R S_{H z}^{1} \gtreqless y^{1} \Leftrightarrow \frac{\varepsilon_{z}^{1}}{\varepsilon_{z}^{2}} \lesseqgtr \frac{\varepsilon_{H}^{1}}{\varepsilon_{H}^{2}},
$$

which from (9) implies

$$
\theta^{1} \lesseqgtr 0 \Leftrightarrow \frac{\varepsilon_{z}^{1}}{\varepsilon_{z}^{2}} \lesseqgtr \frac{\varepsilon_{H}^{1}}{\varepsilon_{H}^{2}} .
$$

Consequently, the $(H, z)$ tradeoff is distorted upward $(\theta<0)$ when the elasticity of disutility for health dominates the one of retirement i.e., when $\varepsilon_{z}^{1} / \varepsilon_{z}^{2}<\varepsilon_{H}^{1} / \varepsilon_{H}^{2}$.

To understand this result, let us return to Figure 1 which illustrates an individual's choice of $(z, H)$. Recall that for a given pair $(y, I)$ maximizing utility is equivalent to minimizing $R$. If $\varepsilon_{z}^{1} / \varepsilon_{z}^{2}<\varepsilon_{H}^{1} / \varepsilon_{H}^{2}$, the slope of the $\bar{R}$ curve at any given point $(z, H)$ is smaller for individual 1 than for individual 2. This implies that individual 1 prefers 
relatively more health than individual 2 for a given $(y, I)$ pair. In other words, the $(z, H)$ pair chosen by individual 1 lies to the north east of the point chosen by individual 2 on the life cycle income line $H=I+z y$. Consequently, the self-selection constraint can be relaxed by distorting individual 1's $(z, H)$ tradeoff towards higher health expenditures and a longer career. In the opposite case, when $\varepsilon_{z}^{1} / \varepsilon_{z}^{2}>\varepsilon_{H}^{1} / \varepsilon_{H}^{2}$, the $(z, H)$ pair chosen by individual 1 lies to the south west of the point chosen by individual 2 . Then, the way to relax the self selection constraint is to induce individuals 1 to distort their $(z, H)$ tradeoff toward shorter career and lower health expenditures.

\section{Publicly observable anonymous transactions}

\subsection{Policy instruments and individual choices}

We now assume that individual levels of $H$ are not publicly observable. Consequently, the non-linear subsidy considered in the previous section is no longer feasible. What is available is information on anonymous transactions so that a linear subsidy (or tax) on $H$ remains feasible. We characterize the utilitarian allocation that is constrained by both the standard self-selection constraint and the linearity of the subsidy on health services. To do this, we characterize an optimal revelation mechanism which consists of a set of type specific bundles of after tax and before tax incomes and retirement ages $\left(D^{i}, y^{i}, z^{i}\right), i=1,2$, and of the subsidy rate, or equivalently the consumer price of $H$ (same for everyone), $p$. A complete solution to the optimal tax problem then requires only the design of an implementing general income tax function. ${ }^{11}$

To proceed further, it is necessary to consider the optimization problem of an individual for a given mechanism $\left(p, D^{i}, y^{i}, z^{i}\right)$. This is necessitated by the fact that the mechanism determines individual consumption of health service only indirectly, namely through their (consumer) price. Individuals demand for health services $H^{i}(p, D, y, z)$ is determined by solving

$$
\underset{H}{\operatorname{Max}} u(D-p H)-V\left(\frac{y}{w}\right) R(z, H) .
$$

\footnotetext{
${ }^{11}$ For consistency, it is assumed that individual consumption levels of the numeraire good $c^{i}$ are not observable either. This is because the budget constraint implies $c^{i}=D^{i}-p H^{i}$ so that when $c^{i}$ is observable (and with $D^{i}$ set by the government), $H^{i}$ would effectively be known.
} 
The first-order condition is given by

$$
-p u^{\prime}(c)-V(l) R_{H}(z, H)=0 .
$$

The following lemma, established in the appendix, will be useful to study the second best solution:

Lemma 1 Under Assumption 1 we have for any given vector of parameters $(p, D, y, z)$

$$
H^{2}(p, D, y, z) \leq H^{1}(p, D, y, z)
$$

In words, this lemma states that at any given point, i.e., when all observable variables (pre-tax income, after-tax income, retirement age and price of health services) are set at the same level, then type 2 individuals consume less health services than type 1 individuals. Observe that the inequality is strict when $w^{2}>w^{1}$ or when Assumption 1 holds with strict inequality.

\subsection{Second best optimum}

The utilitarian second best solution is determined by solving the following problem

$$
\begin{aligned}
& \max _{D^{i}, y^{i}, z^{i}, p} \sum_{i} f^{i}\left[u\left(D^{i}-p H^{i}\left(\left(p, D^{i}, y^{i}, z^{i}\right)\right)\right)-V\left(\frac{y^{i}}{w^{i}}\right) R^{i}\left(z^{i}, H^{i}\left(p, D^{i}, y^{i}, z^{i}\right)\right)\right] \\
& +\mu\left[\sum_{i} f^{i}\left(y^{i} z^{i}-D^{i}+(p-1) H^{i}\left(p, D^{i}, y^{i}, z^{i}\right)\right]\right. \\
& +\lambda\left[u\left(D^{2}-p H^{2}\left(p, D^{2}, y^{2}, z^{2}\right)\right)-V\left(\frac{y^{2}}{w^{2}}\right) R^{2}\left(z^{2}, H^{2}\left(p, D^{2}, y^{2}, z^{2}\right)\right)\right. \\
& \left.\quad-u\left(D^{1}-p H^{2}\left(p, D^{1}, y^{1}, z^{1}\right)\right)+V\left(\frac{y^{1}}{w^{2}}\right) R^{2}\left(z^{1}, H^{2}\left(p, D^{1}, y^{1}, z^{1}\right)\right)\right] .
\end{aligned}
$$

This problem differs from the specification in the non linear case (11) in that $H^{i}$ are controlled only indirectly. This has the most crucial impact on the self-selection constraint in that the mimicking individual no longer has the same consumption vector as the mimicked individual. Denoting $\bar{H}^{2}=H^{2}\left(p, D^{1}, y^{1}, z^{1}\right)$ and $\bar{c}^{2}=D^{1}-p \bar{H}^{2}$, the consumption levels of the mimicking individual, and using the envelop theorem to 
differentiate the (indirect) utility levels in the incentive constraint, FOC with respect to $D^{1}, D^{2}$ and $p$ are given by

$$
\begin{aligned}
& f^{1} u^{\prime}\left(c^{1}\right)+\mu f^{1}\left[(p-1) H_{D}^{1}-1\right]-\lambda u^{\prime}\left(\bar{c}^{2}\right)=0 \\
& f^{2} u^{\prime}\left(c^{2}\right)+\mu f^{2}\left[(p-1) H_{D}^{2}-1\right]+\lambda u^{\prime}\left(c^{2}\right)=0 \\
& -\sum_{i} f^{i} u^{\prime}\left(c^{i}\right) H^{i}+\mu \sum_{i} f^{i}\left[H^{i}+(p-1) H_{p}^{i}\right]+\lambda\left[\bar{H}^{2} u^{\prime}\left(\bar{c}^{2}\right)-H^{2} u^{\prime}\left(c^{2}\right)\right]=0,
\end{aligned}
$$

Combining these first order conditions yields the following expression for the optimal subsidy rate on health expenditures ${ }^{12}$

$$
p-1=\left[\frac{\lambda u^{\prime}\left(\bar{c}^{2}\right)\left(H^{1}-\bar{H}^{2}\right)}{\mu \sum_{i} \widetilde{H}_{p}^{i}}\right]
$$

where $\widetilde{H}_{p}^{i}<0$ is the derivative with respect to $p$ of the compensated (Hicksian) demand for $H^{i}$.

The denominator measures the efficiency cost (deadweight loss) associated with a (positive or negative) subsidy on health. Health is subsidized, $p<1$ if $H^{1}\left(p, D^{1}, y^{1}, z^{1}\right)>$ $H^{2}\left(p, D^{1}, y^{1}, z^{1}\right)=\bar{H}^{2}$, i.e., when the mimicked individual 1 has a larger demand of $H$ than the mimicker. This is the usual condition (see, e.g., Cremer et al. (2001)) for the desirability of (positive or negative) commodity taxation. The nice feature in our setting is that from Lemma 1, we know that under Assumption 1 we necessarily have $p \leq 1$. Furthermore, when $w^{2}>w^{1}$ or when Assumption 1 holds with strict inequality, we have necessarily a subsidy on health expenditures $p<1 .{ }^{13}$ Observe that unlike in the previous section this subsidy is now given to all individuals. Needless to say that this allocation yields a lower level of welfare than the one achieved when individual levels of $H$ are observable.

\section{Public provision of health services}

So far we have concentrated on linear or nonlinear subsidies on health expenditures. We have shown that under plausible conditions such subsidies are part of the optimal policy

\footnotetext{
${ }^{12}$ To obtain (30) we sum up equation (29) with equation (27) times $H_{1}$ and equation (28) times $H_{2}$ and then solve with respect to $p$.

${ }^{13}$ We get $\mathrm{p}=1$ when $w^{1}=w^{2}$ and $R_{1 H}(z, H)=R_{2 H}(z, H)$
} 
mix. They constitute a useful instrument even when there is a well designed nonlinear income tax. In addition to subsidies, public provision of health services is a widespread form of government intervention in the health sector. Put differently, health services are often provided in-kind rather than through subsidization of market provision. We shall now examine if this additional instrument can be useful in our setting. Again we restrict our attention to health service as reducing the difficulty of working long.

The answer depends first and foremost on the information structure. When nonlinear subsidies are feasible as in section 3, there is of course no room for further welfare improvements through public provision; see Cremer and Gahvari (1997) for a detailed discussion. Any Pareto efficient self selection allocation is readily implementable through the tax and subsidy system. Consequently, there is no role for in kind transfers in that setting.

When only anonymous transactions are observable (so that subsidies are restricted to be linear), we can no longer rule out in-kind transfers on an a priori basis. Assuming that publicly provided health services cannot be traded on a secondary market, in-kind transfers of health services do provide partial information on individual consumption levels. This extra information can potentially be welfare improving.

Public provision can potential be uniform or non uniform (as long as the self-selection constraints are satisfied). He we shall concentrate on uniform public provision which is the simplest form. We shall examine whether providing $g$ units of $H$ "free of charge" (i.e., financed by a uniform lump-sum tax equal to $p g$ ) to everyone can bring about a welfare improvement. The benchmark scenario we use is the optimal solution in Section 4 (optimal non linear income tax and linear subsidy).

Our analysis is closely inspired by Cremer and Gahvari (1997) who show that under a condition on the relative consumption levels of the different types (including the mimicker), uniform public provision can relax an otherwise binding incentive constraint and hence be welfare improving. In our context, Cremer and Gahvari's Condition 1 writes as

$$
H^{2}\left(p, D^{1}, y^{1}, z^{1}\right)=\bar{H}^{2}<\min \left[H^{1}\left(p, D^{1}, y^{1}, z^{1}\right), H^{2}\left(p, D^{2}, y^{2}, z^{2}\right)\right]
$$


In words, the (hypothetical) consumption level of the mimicking individual $\bar{H}^{2}$ is smaller than the actual consumption levels of both types of individuals. We know from Lemma 1 that $\bar{H}^{2}<H^{1}\left(p, D^{1}, y^{1}, z^{1}\right)$ hold when Assumption 1 is satisfied with strict inequality. Condition (31) then reduces to

$$
\bar{H}^{2}<H^{2}\left(p, D^{2}, y^{2}, z^{2}\right)
$$

This condition says that individual 2's consumption of health services must be smaller when he mimics individual 1, than when he chooses his own consumption bundle. Intuitively, one would expect this condition to be satisfied. Assuming that $\left(D^{2}, y^{2}, z^{2}\right)>$ $\left(D^{1}, y^{1}, z^{1}\right)$ is satisfied the mimicking individual retires earlier, works less and has a lower disposable income than individual 2. If $H^{2}$ is an increasing function of its last three arguments, these three factors then contribute to lowering the level of health services consumed by the mimicking individual. From the first and second order conditions of an individual's problem (24), one easily shows that this is indeed the case as long as $R_{H z}<0$. This condition (which we can also write $-R_{H z}>0$ ) states roughly speaking that health expenditures are the more "productive" (improving the health status) the later the individual retires.

Following the result stated by Proposition 3 in Cremer and Gahvari (1997), we can then conclude that under Assumption 1 and if the solution in Section 4 satisfies (32), a public provision of health services at level

$$
g=\min \left[H^{1}\left(p, D^{1}, y^{1}, z^{1}\right), H^{2}\left(p, D^{2}, y^{2}, z^{2}\right)\right]-\varepsilon,
$$

with $\varepsilon>0$ and sufficiently small to ensure $g>\bar{H}^{2}$, along with a suitable adjustment in the tax policy, is welfare improving.

To understand the intuition behind this result, assume first that the $g$ units of health services specified by (33) are financed by a uniform lump sum tax at level $p g$. Then, the policy has no impact on actual consumption levels. Consequently, it has no effect on individual utilities and no effect on tax revenue. However, it relaxes the otherwise binding incentive constraint because it imposes a higher than desired level of health expenditures on the mimicker. With the incentive constraint no longer binding, it is 
then possible to adjust the tax policy to bring about an increase in welfare. Roughly speaking this means that some additional redistribution can be achieved and that we move closer to the first-best solution.

\section{Conclusion}

We have studied the design of pension benefits and contributions when an individual's health status (disutility of continued activity) is endogenous and depends on his consumption of health services. Health services were assumed to be privately provided but could be subsidized (in a linear or non linear way, depending on the information structure). In addition, uniform public provision of health services has been considered as an additional policy instrument, to supplement the optimal subsidy (if any). The following main results were obtained. First, the result obtained in our earlier papers under the assumption of an exogenous health status were shown to be robust in the sense that they continue to hold when the health status is endogenous and depends on the consumption of health services. In particular, the existence of redistributive objectives under asymmetric information continues to call for pension benefit formulas which induce early retirement (at least for some types of individual). Second, whatever the specific information structure considered (individual levels or anonymous transactions) for health services, a subsidization of health expenditures was shown to obtain under fairly plausible assumptions. Third, when the information structure permits only linear subsidies, the case for uniform public provision of health services (which can be supplemented but not resold) has appeared to be quite strong.

The model used in this paper deals with the issue of retirement and health at the time of retirement. It could be used in other settings where individuals can influence the level of a characteristic that is not publicly observable; for instance, the disutility of "daily" labor or the earning capacity. 


\section{References}

[1] Cremer, H. and F. Gahvari, (1997), In-kind transfers, self-selection and optimal tax policy, European Economic Review, 47, 97-114.

[2] Cremer, H., J-M. Lozachmeur and P. Pestieau, (2004a), Social security, retirement age and optimal income taxation, Journal of Public Economics, 88, 2259-2281.

[3] Cremer, H., J-M. Lozachmeur and P. Pestieau, (2004b), Optimal retirement and disability benefits with audit, Finanzarchiv, 60 .

[4] Cremer, H., J-M. Lozachmeur and P. Pestieau, (2004c), Disability testing and retirement, mimeo.

[5] Gruber, G. and D. Wise, (1999), eds., Social Security and Retirement around the World, The Chicago University Press, Chicago.

[6] Gruber, G., (2000), Disability insurance benefits and labor supply, Journal of Political Economy, 108, 1162-1183. 


\section{A Appendix}

\section{A.1 Proof of Lemma 1}

Rewrite $R^{i}(z, H)$ as $R\left(z, H, \alpha^{i}\right)$ with $d R / d \alpha^{i}>0$ so that individual 1 with bad health is characterized by $\alpha^{1} \geq \alpha^{2}$. Note that Assumption 1 ensures that $-d R_{H}\left(z, H, \alpha^{i}\right) / d \alpha^{i}>$ 0 . The FOC (25) can be rewritten as:

$$
-p u^{\prime}(D-p H)-V\left(\frac{y}{w}\right) R(z, H, \alpha)=0
$$

where subscripts have been deleted.

This equation defines $H^{i}(p, D, y, z)=H\left(w^{i}, \alpha^{i} ; p, D, y, z\right)$ with derivatives:

$$
\begin{aligned}
\frac{\partial H}{\partial w} & =-\frac{\frac{y}{w^{2}} V^{\prime}\left(\frac{y}{w}\right) R_{H}(z, H, \alpha)}{\Delta} \\
\frac{\partial H}{\partial \alpha} & =\frac{V\left(\frac{y}{w}\right) \frac{\partial R_{H}(z, H, \alpha)}{\partial \alpha}}{\Delta},
\end{aligned}
$$

where $\Delta<0$ is the second order derivative of the left hand side of (34) with respect to $H$. Consequently, we have $\partial H / \partial w<0$ and $\partial H / \partial \alpha>0$ so that $H^{2}(p, D, y, z) \leq$ $H^{1}(p, D, y, z)$. 